\title{
Impaired synthesis contributes to diabetes-induced decrease in liver glutathione
}

\author{
ANNA LISA FURFARO ${ }^{1}$, MARIAPAOLA NITTI ${ }^{1}$, BARBARA MARENGO $^{2}$, \\ CINZIA DOMENICOTTI ${ }^{1}$, DAMIANO COTTALASSO ${ }^{1}$, UMBERTO MARIA MARINARI ${ }^{1}$, \\ MARIA ADELAIDE PRONZATO ${ }^{1}$ and NICOLA TRAVERSO ${ }^{1}$ \\ ${ }^{1}$ Department of Experimental Medicine, Section of General Pathology, University of Genova, Genova; \\ ${ }^{2}$ Giannina Gaslini Institute, Genova, Italy
}

Received October 3, 2011; Accepted November 17, 2011

DOI: $10.3892 / \mathrm{ijmm} .2012 .915$

\begin{abstract}
Diabetes-induced glutathione (GSH) decrease is usually ascribed to GSH oxidation. Here we investigate, in streptozotocin-treated rats, if impairment of GSH synthesis contributes to GSH decrease in diabetic liver, and if antioxidant treatments can provide protection. Diabetic rats were divided into 3 groups: untreated diabetic rats (UD); N-acetylcysteine (NAC)-treated diabetic rats; taurine (TAU)-treated diabetic rats; a group of non-streptozotocin-treated rats was used as control (CTR). All rats were sacrificed at 40 weeks of age. Diabetes induced hepatic glutathione decrease, but oxidized glutathione (GSSG) did not increase significantly. Accumulations of cysteine and cysteinyl-glycine in UD suggest respectively decreased glutathione synthesis and increased loss through the plasma membrane with subsequent degradation. Decreased expression of $\gamma$-glutamyl-cysteine synthetase in UD is consistent with repressed GSH synthesis. Moreover, diabetes caused increase of GSSG/GSH ratio and induction of heme oxygenase-1, both signs of oxidative stress. Supplementation with NAC or TAU resulted in amelioration of glutathione levels, probably depending on antioxidant activity, more efficient glutathione synthesis and decreased GSH loss and degradation. In conclusion, impaired synthesis and increased loss and degradation of GSH appear to contribute to a decrease in GSH levels
\end{abstract}

Correspondence to: Dr Nicola Traverso, Department of Experimental Medicine, Section of General Pathology, University of Genova, Via LB Alberti 2, I-16132 Genova, Italy

E-mail: nicola.traverso@unige.it; nictraverso@yahoo.it

Abbreviations: AGE, advanced glycation end-products; CTR, control; Cys-Gly, cysteinyl-glycine; FDNB, 1-fluoro-2,4-dinitrobenzene; gGCS, $\gamma$-glutamyl-cysteine synthetase; HS, heavy subunit; LS, light subunit; gGT, $\gamma$-glutamyl-transpeptidase; GHb, glycated hemoglobin; GS, glutathione synthetase; GSH, glutathione; GSSG, oxidized glutahione; HO-1, heme oxygenase-1; IAA, iodoacetic acid; NAC, N-acetyl-cysteine; PCA, perchloric acid; TAU, taurine; TBS, Tris-buffered saline; tGSH, total GSH; UD, untreated diabetic rats

Key words: diabetes, glutathione, cysteine, $\mathrm{N}$-acetyl-cysteine, taurine, heme oxygenase in diabetic liver. NAC and TAU are able to partially protect from oxidative stress and GSH decrease, while enhancing GSH synthesis and restricting GSH loss.

\section{Introduction}

Oxidative stress is widely considered as one of the main mechanisms involved in the pathogenesis of chronic complications of diabetes, although its role is under constant discussion (1-6). Several studies have underlined the participation of oxidative mechanisms in the generation of some advanced glycation end-products (AGE) (7), which are considered responsible for protein alterations leading to diabetic sequelae (8). Moreover, signs of increased oxidative stress in diabetes have been recognized in the plasma $(9,10)$ and inside the cells $(10,11)$; however, studies on the content of antioxidant enzymes have yielded contrasting results (12-15), probably owing to differences in diabetes models, diabetes duration and the organs evaluated.

Glutathione (GSH) is the main cellular thiol participating in cellular redox reactions, and the liver is the main site of GSH synthesis (16). Several studies have shown that GSH content is decreased in various tissues of diabetic organisms $(13,14,17,18)$; this could be the cause and consequence of increased oxidative stress. Decrease in glutathione levels has been frequently reported in diabetes, and could occur through decreased synthesis, increased utilization (consumption or degradation) or a combination of both (19).

Synthesis of GSH depends essentially on two enzymes acting sequentially [ $\gamma$-glutamyl-cysteine synthetase (gGCS), EC 6.3.2.2, and glutathione synthetase (GS), EC 6.3.2.3], but only the first is believed to be rate-determinant; availability of cysteine is also an important limiting factor (20).

Consumption of GSH through oxidation is implied or overtly indicated by several authors as the cause of glutathione decrease in diabetes; however, not all authors have measured oxidized glutathione (GSSG) (21-26); the measure of GSSG/ $\mathrm{GSH}$ ratio is anyway fundamental to reveal the existence of oxidative stress (27). It is also to be kept in mind that GSSG can be extruded from the cell, preventing its increase inside the cells during GSH oxidation (28-30). More complex results are sometimes presented, indicating different GSH metabolism in different tissues and subcellular compartments (31). 
However, besides oxidation, GSH can be consumed through other pathways, such as conjugation (spontaneous or catalyzed by glutathione S-transferase) (32), glutathionylation of proteins (16) and transport (or loss) through the plasma membrane with possible subsequent peptidolysis by $\gamma$-glutamyl-transpeptidase (gGT), which converts GSH in aminoacidic compounds able to be taken up again by cells; this mechanism permits transport or exchange of GSH also between distant organs $(29,30,33)$.

In the present study, we investigated the possible reasons for the GSH decrease in diabetic liver, we measured the levels of oxidized glutathione and the expression of heme oxygenase-1 (HO-1) as signs of oxidative stress; moreover, we evaluated cysteine, as a GSH precursor, cysteinyl-glycine (Cys-Gly) as a GSH degradation product, and the expression of the key enzyme of GSH synthesis (gGCS), in order to obtain information about GSH synthesis and degradation. Finally, we supplemented the diabetic rats with the antioxidant substances [N-acetyl-cysteine (NAC), a known antioxidant and precursor of glutathione (34-36), and taurine, a substance ascribed with various potentially beneficial effects (37-39)], in order to clarify if antioxidant supplementation can revert or attenuate the effects of diabetes on glutathione metabolism.

\section{Materials and methods}

Animals. Male Wistar rats $(\mathrm{n}=26)$ purchased from Harlan Italy Srl (Milan, Italy) were randomly housed in pairs and fed a standard diet (Piccioni Spa, Milan, Italy) ad libitum. A group of control rats $(\mathrm{CTR}, \mathrm{n}=8)$ was left untreated. At 12 weeks of age, the remaining rats were rendered diabetic by means of a single i.p. injection of streptozotocin $(60 \mathrm{mg} / \mathrm{kg})$, freshly dissolved in sterile citrate buffer ( $\mathrm{pH} 4.5)$ (40-42). Glucose concentration in blood drawn from a tail vein was determined by means of reactive strips and read with a reflectometer (both from Glucotrend $2^{\circledR}$, Boehringher Mannheim Italia Spa, Milan, Italy). Stable blood glucose levels over $11 \mathrm{mmol} / \mathrm{l}$ were considered to indicate the onset of diabetes mellitus (DM).

The diabetic rats were randomly assigned to the following three groups, 6 rats for each group: i) untreated diabetic rats (UD group); ii) rats treated with NAC (300 mg/rat/day) (NAC group); and iii) rats treated with taurine (TAU) $(2.5 \mathrm{~g} / \mathrm{rat} / \mathrm{day})$ (TAU group). NAC and TAU were administered in the drinking water (39,43-45). All rats were maintained at $22-24^{\circ} \mathrm{C}$ with $12-\mathrm{h}$ dark/light cycles, in accordance with common procedures for good animal care. Blood glucose levels were measured daily until diabetes development and then once a week during the entire period of observation. The rats were sacrificed at 40 weeks of age (about 6 months after the onset of diabetes) by bleeding under anesthesia with i.p. injection of sodium pentobarbital $(65 \mathrm{mg} / \mathrm{kg})$. Glycated hemoglobin $(\mathrm{GHb})$ levels were measured by affinity chromatography (Glycaffin ${ }^{\circledR}$, Isolab, Akron, OH, USA) on blood drawn just before the sacrificing.

The experiments were performed in accordance with the Italian national legislation (Italian animal-good care official rules, N. 116/92).

HPLC analyses. Specimens of the liver were immediately processed for the measurement of GSH, GSSG, cysteine and Cys-Gly in HPLC by the method of Fariss and Reed (46). Briefly, specimens of liver were homogenized and precipitated with perchloric acid (PCA) (10\% final); thiol groups were blocked with iodoacetic acid (IAA) at alkaline $\mathrm{pH}$; analytes were then converted to 2,4-dinitrophenyl derivatives by overnight derivatization with $1 \%$ 1-fluoro-2,4-dinitrobenzene (FDNB) at $4^{\circ} \mathrm{C}$ in the dark. Quantitative determination of the derivatized analytes was performed in HPLC; the HPLC system was equipped with two Waters 510 pumps, with a Waters Spherisorb $5 \mu \mathrm{m} \mathrm{NH} \mathrm{NH}_{2}$ column and a Kontron $332 \mathrm{UV}$ detector set at $365 \mathrm{~nm}$; the flow rate was $1.5 \mathrm{ml} / \mathrm{min}$. The mobile phase was maintained at $80 \% \mathrm{~A}(80 \%$ methanol) and $20 \% \mathrm{~B}$ (0.5 M sodium acetate in 64\% methanol) for $5 \mathrm{~min}$, followed by a 10-min linear gradient to $1 \% \mathrm{~A}$ and $99 \% \mathrm{~B}$; the mobile phase was maintained at 99\% B until GSSG eluted. Total GSH (tGSH) content was calculated in the chromatograms by adding GSH + 2GSSG, and expressed in GSH equivalents (16).

RT-PCR studies. Expression of mRNA of HO-1, of the heavy and light subunit of $\gamma$-glutamyl-cysteine synthetase (gGCS-HS and gGCS-LS, respectively) and of $\beta$-actin, as a house keeping gene, were evaluated by semiquantitative RT-PCR.

Total-RNA was extracted using TRIzol reagent (Invitrogen, Milano, Italy) according to the manufacturer's instructions. One microgram of total-RNA was reverse transcribed into cDNA by SuperScript ${ }^{\mathrm{TM}}$ II reverse transcriptase (Invitrogen) in the presence of polyadenylated oligonucleotides.

Amplification of cDNA by polymerase chain reaction was performed using PCR Master mix (Fermentas, Milano, Italy) and specific primers (47-49), synthesized by TIB Molbiol (Genova, Italy). The amplification products were separated by electrophoresis on $1.5 \%$ agarose ethidium bromide gel and analyzed by means of the Gel Doc 2000 densitometer (BioRad, Milano, Italy) through the 'Molecular Analyst' software (Bio-Rad).

Western blot analyses. For Western blotting (WB) analysis, liver tissue $(150 \mathrm{mg})$ was homogenized in $1.5 \mathrm{ml}$ cold phosphate buffer ( $5 \mathrm{mM}$, pH 7.4) containing $0.1 \%$ Triton X-100 and protease inhibitor cocktail (Sigma-Aldrich, Milano, Italy). The homogenate was clarified by centrifugation at $10,000 \mathrm{x} \mathrm{g}$ for $25 \mathrm{~min}$ at $4^{\circ} \mathrm{C}$. The supernatant was used for the determination of protein concentration (BCA, Pierce, USA) and for Western blotting for gGCS (heavy and light subunit), HO-1 and actin. Protein extracts were separated by SDS-PAGE in $6.5 \%(\mathrm{v} / \mathrm{v})$ stacking and $10 \%(\mathrm{v} / \mathrm{v})$ separation gels. Separated proteins were then transferred to a PVDF membrane (Amersham Biosciences, Piscataway, NJ, USA). After a 1 h blocking step with 5\% milk in $20 \mathrm{mM}$ TBS-0.05\% Tween-20 (pH 7.5), the membranes were incubated for $1 \mathrm{~h}$ at room temperature with rabbit polyclonal antibodies $(1: 20,000)$ raised against rat gGCS heavy and light subunits, which were generously provided as a gift by Dr T.J. Kavanagh (University of Washington, Seattle, USA) or with rabbit polyclonal antibodies $(1: 5,000)$ raised against rat HO-1 (Stressgen Biotechnologies, San Diego, CA, USA). The membranes were washed three times, incubated for $1 \mathrm{~h}$ at room temperature with goat anti-rabbit IgG antibody conjugated to horseradish peroxidase (Amersham Biosciences), and again washed three times. Chemiluminescence was developed using the ECL-plus substrate (Amersham Biosciences). Bands were detected using the ECL-Hyperfilm (Amersham Biosciences) 


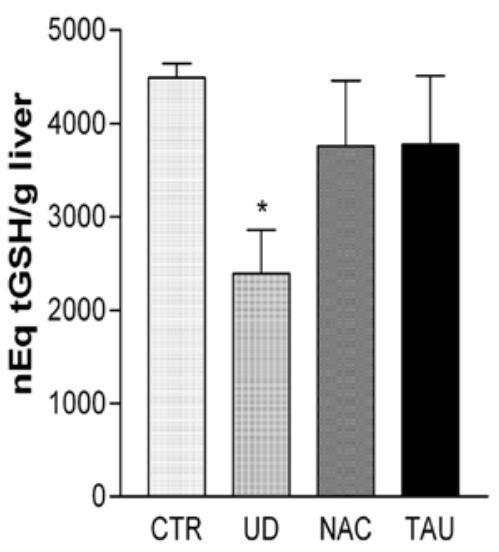

Figure 1. Hepatic total GSH (tGSH) content. Data are expressed as mean \pm SEM. CTR, control group; UD, untreated diabetic rats; NAC, diabetic rats treated with $\mathrm{N}$-acetyl-cysteine; TAU, diabetic rats treated with taurine. * $\mathrm{P}<0.05$ vs. CTR.

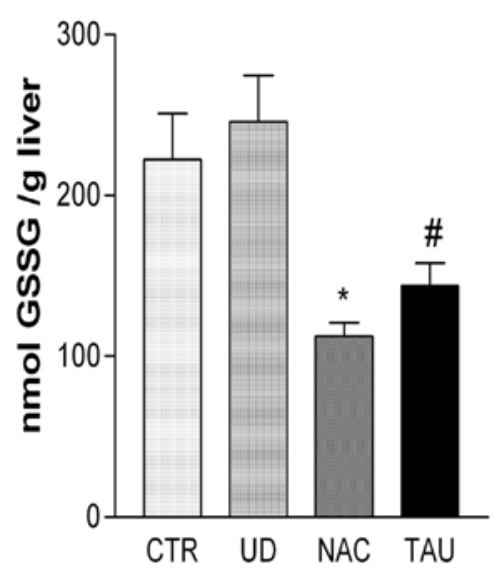

Figure 2. Hepatic oxidized glutathione (GSSG) content. Data are expressed as mean \pm SEM. CTR, control group; UD, untreated diabetic rats; NAC, diabetic rats treated with $\mathrm{N}$-acetyl-cysteine; TAU, diabetic rats treated with taurine. ${ }^{*} \mathrm{P}<0.01$ vs. CTR and vs. UD; ${ }^{*} \mathrm{P}<0.05$ vs. CTR and vs. UD.

and quantification of chemiluminescence was performed with the Gel Doc 2000 densitometer (Bio-Rad, Hercules, CA, USA). To compare protein loading, membranes were reprobed with a mouse monoclonal anti-actin antibody (Sigma).

Statistical analyses. Results were expressed as mean \pm SEM. The statistical significance of parametric differences among sets of experimental data was evaluated by ANOVA and the student Newman-Keuls post-test for multiple comparison by the GraphPad Prism program.

\section{Results}

Streptozotocin induced an abrupt increase in blood glucose to $\mathrm{P}$-values $>11 \mathrm{mmol} / \mathrm{l}$. Mean blood glucose in the last 10 weeks of life was lower in NAC-treated $(24.17 \pm 1.11 \mathrm{mmol} / 1 ; \mathrm{P}<0.05$ vs. UD) and TAU-treated $(26.11 \pm 1.67 \mathrm{mmol} / \mathrm{l} ; \mathrm{P}>0.05 \mathrm{vs}$. UD) diabetic rats than in UD rats $(29.44 \pm 1.33 \mathrm{mmol} / \mathrm{l})$. This observation was paralleled by the total $\mathrm{GHb}$ concentration, with the UD group showing significantly higher mean GHb $(20 \pm 2 \%)$

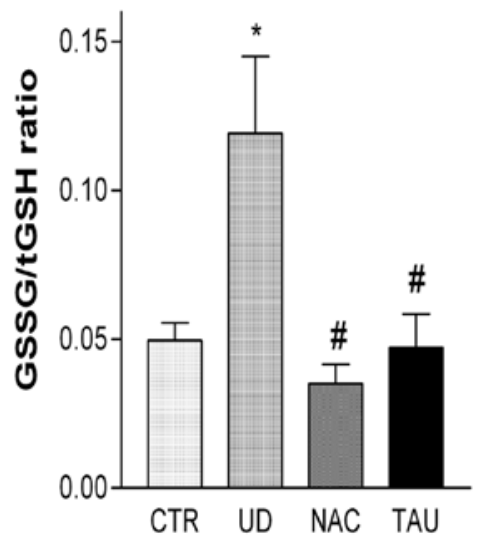

Figure 3. GSSG/tGSH ratio in the liver. Data are expressed as mean \pm SEM. CTR, control group; UD, untreated diabetic rats; NAC, diabetic rats treated with $\mathrm{N}$-acetyl-cysteine; TAU, diabetic rats treated with taurine. ${ }^{*} \mathrm{P}<0.001$ vs. CTR; ${ }^{\#} \mathrm{P}<0.01$ vs. UD.

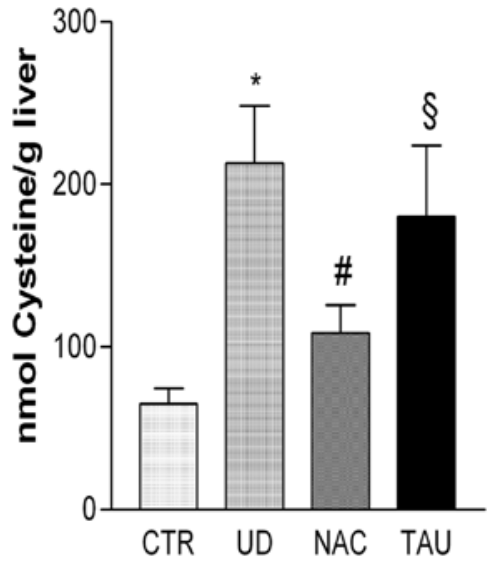

Figure 4. Hepatic cysteine content. Data are expressed as mean \pm SEM. CTR control group; UD, untreated diabetic rats; NAC, diabetic rats treated with $\mathrm{N}$-acetyl-cysteine; TAU, diabetic rats treated with taurine. ${ }^{*} \mathrm{P}<0.01$ vs. CTR; ${ }^{\#} \mathrm{P}<0.05$ vs. UD; ${ }^{\circledR} \mathrm{P}<0.05$ vs. CTR.

values than NAC group $(15 \pm 1.5 \% ; \mathrm{P}<0.01$ vs. $\mathrm{UD})$ or TAU group (16 $\pm 1.5 \%$; $\mathrm{P}<0.01$ vs. UD). CTR mean blood glucose levels in the last 10 weeks of life were $8.06 \pm 0.30 \mathrm{mmol} / \mathrm{l}$ and total GHb levels were $7 \pm 0.5 \%$.

The evaluation in HPLC revealed that diabetes induced a profound decrement in tGSH, which decreased to nearly half of the control value: (UD vs. CTR P<0.05). NAC- and TAU-treated rats had tGSH levels higher than UD, and not significantly different from CTR (Fig. 1).

The evaluation of GSSG in HPLC did not revealed any statistical difference between the UD and CTR rats; however, NAC and TAU were able to decrease GSSG content in comparison to UD treatment (NAC vs. UD P $<0.01$; TAU vs. UD P $<0.05$ ) and to the CTR group (NAC vs. CTR $\mathrm{P}<0.01$ and TAU vs. CTR $\mathrm{P}<0.05$, respectively) (Fig. 2).

In consequence, the GSSG/tGSH ratios were significantly higher in the UD compared to the CTR rats $(\mathrm{P}<0.001)$; in NACand TAU-treated rats this ratio decreased to values not different from CTR, but significantly lower than in UD rats $(\mathrm{P}<0.01)$ (Fig. 3). 


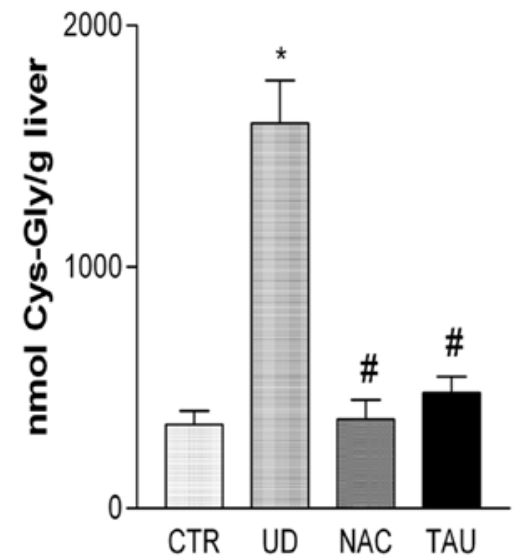

Figure 5. Hepatic cysteinyl-glycine (Cys-Gly) content. Data are expressed as mean \pm SEM. CTR, control group; UD, untreated diabetic rats; NAC, diabetic rats treated with $\mathrm{N}$-acetyl-cysteine; TAU, diabetic rats treated with taurine. ${ }^{*} \mathrm{P}<0.001$ vs. CTR; ${ }^{\mathrm{P}}<0.001$ vs. UD.
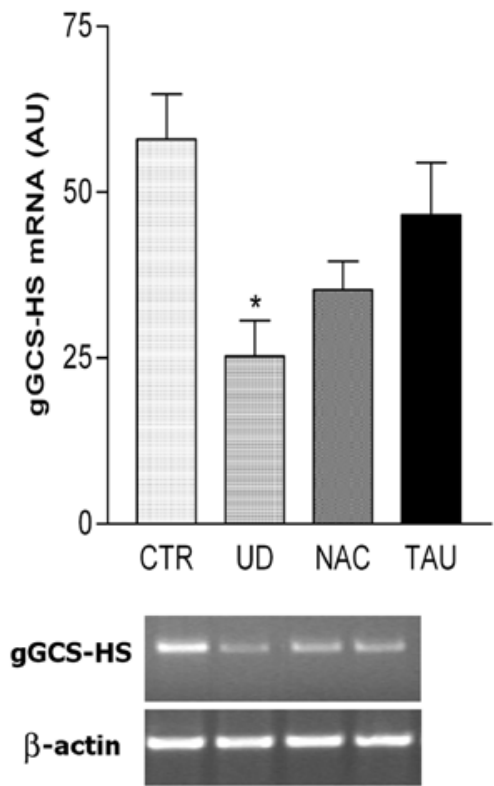

Figure 6. Hepatic mRNA expression of the heavy subunit of $\gamma$-glutamylcysteine synthetase (gGCS-HS). Data are expressed as mean \pm SEM. CTR, control group; UD, untreated diabetic rats; NAC, diabetic rats treated with $\mathrm{N}$-acetyl-cysteine; TAU, diabetic rats treated with taurine. ${ }^{*} \mathrm{P}<0.05$ vs. CTR.

The cysteine content was significantly higher in UD than in CTR rats $(\mathrm{P}<0.01)$. NAC treatment decreased the increment of cysteine seen in UD rats $(\mathrm{P}<0.05)$. TAU treatment did not modify the value induced by diabetes, which remained therefore significantly higher than in the CTR rats $(\mathrm{P}<0.05)$ (Fig. 4).

Cysteinyl-glycine (Cys-Gly) content was dramatically increased in the UD-treated rats in comparison to the CTR group $(\mathrm{P}<0.001)$. NAC and TAU treatments completely protected the rats from the accumulation of Cys-Gly induced by diabetes $(\mathrm{P}<0.001)$, decreasing its content to values not significantly different from CTR (Fig. 5).

RT-PCR analysis of the heavy subunit of gGCS revealed that UD showed a significant decrease of mRNA for this protein in comparison with CTR $(\mathrm{P}<0.05)$; NAC and TAU
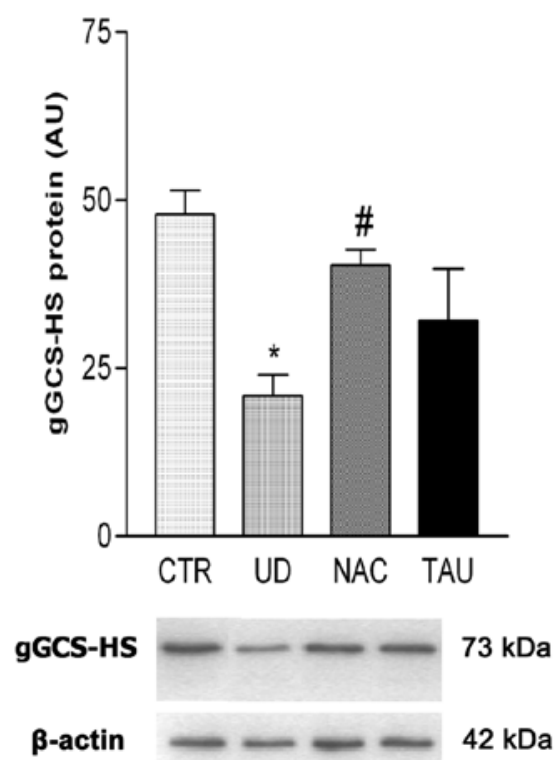

Figure 7. Hepatic protein level of the heavy subunit of $\gamma$-glutamyl-cysteine synthetase (gGCS-HS). Data are expressed as mean \pm SEM. CTR, control group; UD, untreated diabetic rats; NAC, diabetic rats treated with $\mathrm{N}$-acetyl-cysteine; TAU, diabetic rats treated with taurine. ${ }^{*} \mathrm{P}<0.05$ vs. CTR; ${ }^{\#} \mathrm{P}<0.05$ vs. CTR.

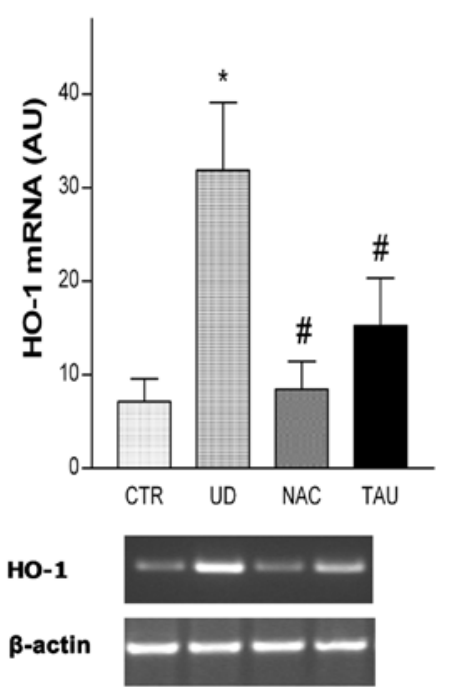

Figure 8. Hepatic mRNA expression of heme oxygenase-1 (HO-1). Data are expressed as mean \pm SEM. CTR, control group; UD, untreated diabetic rats; $\mathrm{NAC}$, diabetic rats treated with $\mathrm{N}$-acetyl-cysteine; TAU, diabetic rats treated with taurine. ${ }^{*} \mathrm{P}<0.05$ vs. CTR; ${ }^{\#} \mathrm{P}<0.05$ vs. UD.

treatments decreased the difference vs. CTR (Fig. 6). No significant difference among the various groups was noted for the mRNA for the light subunit of gGCS (data not shown).

WB revealed that UD-treated rats had significantly lower levels of gGCS-HS protein in comparison with the CTR group $(\mathrm{P}<0.05)$; NAC treatment was able to afford protection from this decrease $(\mathrm{P}<0.05$ vs. UD); TAU treatment resulted in a value higher than UD, but the difference was not significant (Fig. 7). WB did not evidence any significant difference in the levels of gGCS-LS among the groups.

RT-PCR analysis revealed a strong overexpression of HO-1 mRNA for UD-treated rats in comparison to CTR $(\mathrm{P}<0.05)$; 
NAC and TAU protected the animals from this overexpression $(\mathrm{P}<0.05)$ (Fig. 8). Analysis of $\mathrm{HO}-1$ protein expression revealed analogous results (data not shown).

\section{Discussion}

The widely recognized decrease in GSH in diabetic tissues $(13,14,17,18)$ has been confirmed in the liver of our experimental model. Moreover, diabetes was able to cause remarkable increment of the GSSG/tGSH ratio, which is a clear sign of increased oxidative stress (27). Moreover, diabetes dramatically increased the expression of HO-1, a well known mechanism of cellular response to oxidative stress (50). Oxidation to GSSG could therefore be considered a factor responsible for GSH depletion, while the GSSG produced during GSH oxidation could be exported outside the cell, thus maintaining intracellular GSSG concentration quite constant (28-30).

However, we examined other possible mechanisms responsible for the observed GSH decrease. Firstly, we investigated if diabetes induced a decrease in GSH synthesis. In our experimental model, diabetes was able to cause an increase in cysteine content; we suggest that cysteine accumulation is due to a diabetes-induced decrease in GSH synthesis (20). Cysteine accumulation should enhance GSH synthesis, but we hypothesize that in this context cysteine accumulation is not enough to overcome the impairment of GSH synthesis. The hypothesis of diabetes impairing GSH synthesis is reinforced by the fact that UD rats showed decreased expression of gGCS-HS, the catalytic moiety of the key enzyme for GSH synthesis; this decreased expression has been noted previously by Doi et al (51) in the heart of streptozotocin-diabetic rats. Imbalance of insulin equilibrium (52), glycation (53) and oxidative stress with thiol regulation at the cysteine site (54-56) may combine and result in the repression of gGCS-HS expression or activity and GSH synthesis in diabetic rats. A critical element may also be the long term glucose exposure, which has been suggested as a factor able to impair the responsive expression of gGCS (57).

On the other hand, Cys-Gly, an extracellular metabolite of GSH degradation catalysed by gGT (34), accumulated in UD rats; we suggest that this increase could depend on the increased secretion or loss of GSH out of the hepatocytes, followed by GSH degradation. The loss of GSH through the membrane may in turn depend on membrane oxidative alterations due to the diabetes-induced oxidative stress; lipid peroxidation has actually been described to be increased in diabetic rat liver $(11,58,59)$, and peroxidized membrane can exhibit altered permeability $(60,61)$ and transport functions $(62,63)$.

Therefore, our data confirm the existence of GSH decrease and increased oxidative stress in diabetes. However, while oxidation to GSSG is presumably involved in GSH depletion (21-26), our data prompt us to suggest other mechanisms as well, such as synthesis impairment, and leakage from the hepatocytes. In this view, GSH oxidation, impairment of GSH synthesis and GSH loss could be viewed as various and intertwined causes of GSH depletion. Diabetic hepatocytes, GSH-depleted and oxidatively stressed, should then be regarded as particularly susceptible to further damage (e.g. toxic insults).

NAC treatment was able to protect the diabetic rats from the hepatic GSH decrease. Moreover, NAC treatment decreased
GSSG content, and restored the GSSG/tGSH ratio and HO-1 expression to the levels of CTR: these data make us believe that NAC is able to significantly protect diabetic rats from the diabetes-induced oxidative stress; the restored GSH content is likely to be a factor responsible for the protection from oxidative stress; moreover, NAC itself can behave as an antioxidant as well $(34,64,65)$.

In NAC-treated diabetic rats the content of cysteine was significantly lower than in the UD group. We propose that NAC is able to remove the diabetes-induced impairment of GSH synthesis. The observed partial restoration of the levels of gGCS-HS levels is probably one of the crucial reasons for GSH restoration, together with the higher availability of precursor (cysteine derived from NAC) (20); in this way, GSH content can be restored. However, cysteine accumulates in comparison to CTR, reasonably because not all the NAC-derived cysteine is utilized for the synthesis of GSH.

The relation between NAC, gGCS and GSH synthesis is complex. NAC has been both recognized as a cysteine donor (66), able to stimulate gGCS activity, and as an inhibitor of the oxidative stress-induced cascade leading to gGCS transcription (67). Moreover, NAC has been suggested to be able to improve the salvage pathway in which gGT utilizes cystine as the acceptor receiving the $\gamma$-glutamyl moiety from GSH exported from non-hepatocyte cell types. This reaction produces $\gamma$-glutamylcystine, which can be reduced intracellularly to $\gamma$-glutamylcysteine (68).

On the other hand, NAC decreased Cys-Gly content to levels comparable to CTR; this prompts us to suppose that NAC protects hepatocytes from GSH loss through the membrane, which is followed by GSH degradation. Therefore, since NAC protected diabetic rats in parallel from oxidative stress and from Cys-Gly accumulation, we suggest that diabetes-induced GSH leakage occurs and depends on oxidative alterations of the hepatocyte membrane.

In conclusion, NAC probably interferes with various pathological mechanisms in the diabetic rat liver, protecting from oxidative stress, enhancing gGCS activity, facilitating gGCS transcription, restricting GSH loss and maybe stimulating alternative pathways of GSH synthesis.

TAU treatment, similarly to NAC, protected the diabetic rats from hepatic GSH decrease. TAU was able to decrease the GSSG level and to restore the GSSG/tGSH ratio; similar results were obtained by Yao et al (44); moreover, TAU substantially decreased the level of HO-1 expression. These data support an antioxidant role of TAU in diabetic rats, which protects them from the diabetes-induced oxidative stress. The possibility that TAU is able to enhance the reduction of GSSG to GSH has been suggested by Gossai and Lau-Cam (69).

In TAU-treated diabetic rats, cysteine levels were high; since TAU is a product of cysteine degradation through the cysteine dioxygenase pathway (70), it could act as an inhibitor of this pathway, inducing accumulation of cysteine. In this way, more cysteine could be available for GSH synthesis; moreover, TAU provided a mild increase of gGCS-HS expression in comparison to UD. In TAU-treated diabetic rats, the level of Cys-Gly was comparable to that of NAC or CTR; the decreased peptidolytic degradation of GSH could depend on a protection of the membranes from lipoperoxidation, and consequent GSH leakage, in a manner similar to NAC. 
Therefore, we suggest that higher gGCS-HS expression, its activation due to larger cysteine availability and protection from GSH loss are the mechanisms whereby TAU treatment induces GSH restoration in the liver of diabetic rats. Antioxidant and antiglycation properties of TAU (38) may be at the basis of the observed effects on GSH in diabetic liver.

In conclusion, we believe that, although diabetes is able to generate oxidative stress (e.g. increased GSSG/GSH ratio, induced expression of HO-1), hepatic GSH decrease in diabetes is not only caused by GSH oxidation to GSSG, but also by impaired synthesis and increased loss through the hepatocyte membrane, probably related to oxidative damage of the membrane itself. A vicious circle between GSH decrease, oxidative stress and increased loss can be then be generated. $\mathrm{N}$-acetyl-cysteine and taurine are able to provide protection from oxidative stress and GSH decrease, while enhancing GSH synthesis and restricting GSH loss; therefore, these antioxidant substances probably break the vicious circle between GSH decrease and oxidative stress.

\section{Acknowledgements}

We thank Dr Luana Cosso, Dr Stefano Menini and Dr Elena Pesce Maineri for the participation in animal care supervision. We acknowledge the technical assistance of Mr Giuseppe Catalano (DIMES, University of Genova, Italy). This study has been supported by Grants from MIUR 20077S9A32_002, MIUR 2008N9N9KL_002 and the University of Genova.

\section{References}

1. Perez-Matute P, Zulet MA and Martinez JA: Reactive species and diabetes: counteracting oxidative stress to improve health. Curr Opin Pharmacol 9: 771-779, 2009.

2. Scott JA and King GL: Oxidative stress and antioxidant treatment in diabetes. Ann NY Acad Sci 1031: 204-213, 2004.

3. Wiernsperger NF: Oxidative stress as a therapeutic target in diabetes: revisiting the controversy. Diabetes Metab 29: 579-585, 2003.

4. Giugliano D, Ceriello A and Paolisso G: Diabetes mellitus, hypertension, and cardiovascular disease: which role for oxidative stress? Metabolism 44: 363-368, 1995.

5. Baynes JW and Thorpe SR: Role of oxidative stress in diabetic complications: a new perspective on an old paradigm. Diabetes 48: 1-9, 1999

6. Baynes JW: Role of oxidative stress in development of complications in diabetes. Diabetes 40: 405-412, 1991.

7. Fu MX, Knecht KJ, Thorpe SR and Baynes JW: Role of oxygen in cross-linking and chemical modification of collagen by glucose. Diabetes 41 (Suppl 2): S42-S48, 1992.

8. Singh R, Barden A, Mori T and Beilin L: Advanced glycation end-products: a review. Diabetologia 44: 129-146, 2001.

9. Nourooz-Zadeh J, Tajaddini-Sarmadi J, McCarthy S, Betteridge DJ and Wolff SP: Elevated levels of authentic plasma hydroperoxides in NIDDM. Diabetes 44: 1054-1058, 1995.

10. Desco MC, Asensi M, Marquez R, et al: Xanthine oxidase is involved in free radical production in type 1 diabetes: protection by allopurinol. Diabetes 51: 1118-1124, 2002.

11. Traverso N, Menini S, Odetti P, Pronzato MA, Cottalasso D and Marinari UM: Lipoperoxidation in hepatic subcellular compartments of diabetic rats. Free Radic Biol Med 26: 538-547, 1999.

12. Kakkar R, Kalra J, Mantha SV and Prasad K: Lipid peroxidation and activity of antioxidant enzymes in diabetic rats. Mol Cell Biochem 151: 113-119, 1995.

13. Mukherjee B, Mukherjee JR and Chatterjee M: Lipid peroxidation, glutathione levels and changes in glutathione-related enzyme activities in streptozotocin-induced diabetic rats. Immunol Cell Biol 72: 109-114, 1994.
14. Reddi AS and Bollineni JS: Renal cortical expression of mRNAs for antioxidant enzymes in normal and diabetic rats. Biochem Biophys Res Commun 235: 598-601, 1997.

15. Tang Z, Shou I, Wang LN, Fukui M and Tomino Y: Effects of antihypertensive drugs or glycemic control on antioxidant enzyme activities in spontaneously hypertensive rats with diabetes. Nephron 76: 323-330, 1997.

16. Sies H: Glutathione and its role in cellular functions. Free Radic Biol Med 27: 916-921, 1999.

17. Saxena AK, Srivastava P, Kale RK and Baquer NZ: Impaired antioxidant status in diabetic rat liver. Effect of vanadate. Biochem Pharmacol 45: 539-542, 1993.

18. Nwose EU, Jelinek HF, Richards RS and Kerr PG: Changes in the erythrocyte glutathione concentration in the course of diabetes mellitus. Redox Rep 11: 99-104, 2006.

19. Darmaun D, Smith SD, Sweeten S, Sager BK, Welch S and Mauras N: Evidence for accelerated rates of glutathione utilization and glutathione depletion in adolescents with poorly controlled type 1 diabetes. Diabetes 54: 190-196, 2005.

20. Lu SC: Regulation of glutathione synthesis. Mol Aspects Med 30: 42-59, 2009.

21. Chaudhry J, Ghosh NN, Roy K and Chandra R: Antihyperglycemic effect of a new thiazolidinedione analogue and its role in ameliorating oxidative stress in alloxan-induced diabetic rats. Life Sci 80: 1135-1142, 2007.

22. Singh N, Kamath V and Rajini PS: Attenuation of hyperglycemia and associated biochemical parameters in STZ-induced diabetic rats by dietary supplementation of potato peel powder. Clin Chim Acta 353: 165-175, 2005.

23. Anwer T, Sharma M, Pillai KK, Haque SE, Alam MM and Zaman MS: Protective effect of bezafibrate on streptozotocininduced oxidative stress and toxicity in rats. Toxicology 229: 165-172, 2007

24. Murugan P and Pari L: Antioxidant effect of tetrahydrocurcumin in streptozotocin-nicotinamide induced diabetic rats. Life Sci 79: 1720-1728, 2006.

25. Song Y, Wang J, Li Y, et al: Cardiac metallothionein synthesis in streptozotocin-induced diabetic mice, and its protection against diabetes-induced cardiac injury. Am J Pathol 167: 17-26, 2005.

26. de Cavanagh EM, Inserra F, Toblli J, Stella I, Fraga CG and Ferder L: Enalapril attenuates oxidative stress in diabetic rats. Hypertension 38: 1130-1136, 2001.

27. Asensi M, Sastre J, Pallardo FV, et al: Ratio of reduced to oxidized glutathione as indicator of oxidative stress status and DNA damage. Methods Enzymol 299: 267-276, 1999.

28. Keppler D: Export pumps for glutathione S-conjugates. Free Radic Biol Med 27: 985-991, 1999.

29. Homolya L, Varadi A and Sarkadi B: Multidrug resistance-associated proteins: Export pumps for conjugates with glutathione, glucuronate or sulfate. Biofactors 17: 103-114, 2003.

30. Ballatori N, Krance SM, Marchan R and Hammond CL: Plasma membrane glutathione transporters and their roles in cell physiology and pathophysiology. Mol Aspects Med 30: 13-28, 2009.

31. Raza H, Prabu SK, Robin MA and Avadhani NG: Elevated mitochondrial cytochrome P450 2E1 and glutathione S-transferase A4-4 in streptozotocin-induced diabetic rats: tissue-specific variations and roles in oxidative stress. Diabetes 53: 185-194, 2004.

32. Strange RC, Spiteri MA, Ramachandran S and Fryer AA: Glutathione-S-transferase family of enzymes. Mutat Res 482: 21-26, 2001.

33. Griffith OW and Meister A: Glutathione: interorgan translocation, turnover, and metabolism. Proc Natl Acad Sci USA 76: 5606-5610, 1979.

34. Meister A and Anderson ME: Glutathione. Annu Rev Biochem 52: 711-760, 1983.

35. De Flora S, Izzotti A, D'Agostini F and Balansky RM: Mechanisms of $\mathrm{N}$-acetylcysteine in the prevention of DNA damage and cancer, with special reference to smoking-related end-points. Carcinogenesis 22: 999-1013, 2001.

36. Atkuri KR, Mantovani JJ, Herzenberg LA and Herzenberg LA: $\mathrm{N}$-Acetylcysteine - a safe antidote for cysteine/glutathione deficiency. Curr Opin Pharmacol 7: 355-359, 2007.

37. Wright CE, Tallan HH, Lin YY and Gaull GE: Taurine: biological update. Annu Rev Biochem 55: 427-453, 1986.

38. Hansen $\mathrm{SH}$ : The role of taurine in diabetes and the development of diabetic complications. Diabetes Metab Res Rev 17: 330-346, 2001.

39. Odetti P, Pesce C, Traverso N, et al: Comparative trial of N-acetylcysteine, taurine, and oxerutin on skin and kidney damage in long-term experimental diabetes. Diabetes 52: 499-505, 2003.

40. Tesch GH and Allen TJ: Rodent models of streptozotocin-induced diabetic nephropathy. Nephrology (Carlton) 12: 261-266, 2007. 
41. Xu Q, Wells CC, Garman JH, Asico L, Escano CS and Maric C: Imbalance in sex hormone levels exacerbates diabetic renal disease. Hypertension 51: 1218-1224, 2008.

42. Manni L, Florenzano F and Aloe L: Electroacupuncture counteracts the development of thermal hyperalgesia and the alteration of nerve growth factor and sensory neuromodulators induced by streptozotocin in adult rats. Diabetologia 54: 1900-1908, 2011.

43. Xia Z, Kuo KH, Nagareddy PR, et al: N-acetylcysteine attenuates PKCbeta2 overexpression and myocardial hypertrophy in streptozotocin-induced diabetic rats. Cardiovasc Res 73: 770-782, 2007.

44. Yao HT, Lin P, Chang YW, et al: Effect of taurine supplementation on cytochrome P450 2E1 and oxidative stress in the liver and kidneys of rats with streptozotocin-induced diabetes. Food Chem Toxicol 47: 1703-1709, 2009

45. Trachtman H, Futterweit S, Maesaka J, et al: Taurine ameliorates chronic streptozocin-induced diabetic nephropathy in rats. Am J Physiol 269: F429-F438, 1995.

46. Fariss MW and Reed DJ: High-performance liquid chromatography of thiols and disulfides: dinitrophenol derivatives. Methods Enzymol 143: 101-109, 1987.

47. Shibahara S, Muller R, Taguchi $\mathrm{H}$ and Yoshida T: Cloning and expression of cDNA for rat heme oxygenase. Proc Natl Acad Sci USA 82: 7865-7869, 1985.

48. Tian L, Shi MM and Forman HJ: Increased transcription of the regulatory subunit of gamma-glutamylcysteine synthetase in rat lung epithelial L2 cells exposed to oxidative stress or glutathione depletion. Arch Biochem Biophys 342: 126-133, 1997.

49. Morales A, Garcia-Ruiz C, Miranda M, et al: Tumor necrosis factor increases hepatocellular glutathione by transcriptional regulation of the heavy subunit chain of gamma-glutamylcysteine synthetase. J Biol Chem 272: 30371-30379, 1997.

50. Bauer M and Bauer I: Heme oxygenase-1: redox regulation and role in the hepatic response to oxidative stress. Antioxid Redox Signal 4: 749-758, 2002

51. Doi K, Sawada F, Toda G, et al: Alteration of antioxidants during the progression of heart disease in streptozotocin-induced diabetic rats. Free Radic Res 34: 251-261, 2001.

52. Cai J, Huang ZZ and Lu SC: Differential regulation of gammaglutamylcysteine synthetase heavy and light subunit gene expression. Biochem J 326: 167-172, 1997.

53. Fico A, Manganelli G, Cigliano L, et al: 2-deoxy-d-ribose induces apoptosis by inhibiting the synthesis and increasing the efflux of glutathione. Free Radic Biol Med 45: 211-217, 2008.

54. Shi MM, Kugelman A, Iwamoto T, Tian L and Forman HJ: Quinone-induced oxidative stress elevates glutathione and induces gamma-glutamylcysteine synthetase activity in rat lung epithelial L2 cells. J Biol Chem 269: 26512-26517, 1994.

55. Richman PG and Meister A: Regulation of gamma-glutamylcysteine synthetase by non-allosteric feedback inhibition by glutathione. J Biol Chem 250: 1422-1426, 1975.

56. Hicks LM, Cahoon RE, Bonner ER, Rivard RS, Sheffield J and Jez JM: Thiol-based regulation of redox-active glutamatecysteine ligase from Arabidopsis thaliana. Plant Cell 19: 2653-2661, 2007.
57. Urata Y, Yamamoto H, Goto S, et al: Long exposure to high glucose concentration impairs the responsive expression of gammaglutamylcysteine synthetase by interleukin-1beta and tumor necrosis factor-alpha in mouse endothelial cells. J Biol Chem 271: 15146-15152, 1996.

58. Manna P, Das J, Ghosh J and Sil PC: Contribution of type 1 diabetes to rat liver dysfunction and cellular damage via activation of NOS, PARP, IkappaBalpha/NF-kappaB, MAPKs, and mitochondriadependent pathways: Prophylactic role of arjunolic acid. Free Radic Biol Med 48: 1465-1484, 2010.

59. Severcan F, Gorgulu G, Gorgulu ST and Guray T: Rapid monitoring of diabetes-induced lipid peroxidation by Fourier transform infrared spectroscopy: evidence from rat liver microsomal membranes. Anal Biochem 339: 36-40, 2005.

60. Wong-Ekkabut J, Xu Z, Triampo W, Tang IM, Tieleman DP and Monticelli L: Effect of lipid peroxidation on the properties of lipid bilayers: a molecular dynamics study. Biophys J 93: 4225-4236, 2007.

61. Bernotti S, Seidman E, Sinnett D, et al: Inflammatory reaction without endogenous antioxidant response in Caco-2 cells exposed to iron/ascorbate-mediated lipid peroxidation. Am J Physiol Gastrointest Liver Physiol 285: G898-G906, 2003.

62. Sultana R and Butterfield DA: Oxidatively modified GST and MRP1 in Alzheimer's disease brain: implications for accumulation of reactive lipid peroxidation products. Neurochem Res 29: 2215-2220, 2004.

63. Jain SK and Lim G: Pyridoxine and pyridoxamine inhibits superoxide radicals and prevents lipid peroxidation, protein glycosylation, and $\left(\mathrm{Na}^{+}+\mathrm{K}^{+}\right)$-ATPase activity reduction in high glucose-treated human erythrocytes. Free Radic Biol Med 30: 232-237, 2001.

64. Smilkstein MJ, Knapp GL, Kulig KW and Rumack BH: Efficacy of oral $\mathrm{N}$-acetylcysteine in the treatment of acetaminophen overdose. Analysis of the national multicenter study (1976 to 1985). N Engl J Med 319: 1557-1562, 1988.

65. Reliene R and Schiestl RH: Glutathione depletion by buthionine sulfoximine induces DNA deletions in mice. Carcinogenesis 27: 240-244, 2006.

66. De Flora S, Bennicelli C, Camoirano A, et al: In vivo effects of $\mathrm{N}$-acetylcysteine on glutathione metabolism and on the biotransformation of carcinogenic and/or mutagenic compounds. Carcinogenesis 6: 1735-1745, 1985

67. Li HY, Wu SY and Shi N: Transcription factor Nrf2 activation by deltamethrin in PC12 cells: Involvement of ROS. Toxicol Lett 171: 87-98, 2007.

68. Chen Y, Johansson E, Yang Y, et al: Oral N-acetylcysteine rescues lethality of hepatocyte-specific Gclc-knockout mice, providing a model for hepatic cirrhosis. J Hepatol 53: 1085-1094, 2010.

69. Gossai D and Lau-Cam CA: The effects of taurine, taurine homologs and hypotaurine on cell and membrane antioxidative system alterations caused by type 2 diabetes in rat erythrocytes. Adv Exp Med Biol 643: 359-368, 2009.

70. Stipanuk MH, Ueki I, Dominy JE Jr, Simmons CR and Hirschberger LL: Cysteine dioxygenase: a robust system for regulation of cellular cysteine levels. Amino Acids 37: 55-63, 2009. 